\title{
Movilidad urbana en la central de autobuses de la metrópoli de Guadalajara, México: entropía en conectividad y transferencia de usuarios*
}

\author{
Urban mobility at the bus station in the metropolis of Guadalajara, Mexico: entropy in connectivity \\ and user transfer
}

\section{Historial del artículo}

Recibido:

15 de septiembre 2019.

Aceptado:

3 de diciembre de 2019.

\section{Mario González Pérez ${ }^{\mathrm{a}}$, Mauricio Camarena González ${ }^{\mathrm{b}}$, Esmeralda Brito Cervantes ${ }^{\mathrm{c}}$}

${ }^{a}$ Doctor en Ciudad, Territorio y Sustentabilidad, División de Ingenierías e Innovación Tecnológica, Centro Universitario de Tonalá, Universidad de Guadalajara, México. Correo electrónico: inge_united@hotmail.com

${ }^{\mathrm{b}}$ Maestro en Movilidad Urbana, Transporte y Territorio, Centro Universitario de Tonalá, Universidad de Guadalajara, México. c Doctor en Negocios y Estudios Económicos, Coordinación de la Maestría en Gestión de Gobiernos Locales, Centro Universitario de Tonalá, Universidad de Guadalajara, México.

\section{Palabras clave}

Conectividad de autobuses entropía, movilidad urbana, transferencia de usuarios

\section{Keywords}

Bus connectivity, entropy, urban mobility, user transfer

\begin{abstract}
Resumen
Los sistemas urbanos están estructurados a través de una compleja red inducida de conductos comunicantes. Estas vías de acceso suelen presentar obstrucciones y colapsos en función de la saturación del modo utilizado en el transporte de la materia y energía vivas. Asimismo, en ciertos nodos del sistema, existen procesos de conectividad y transferencia de flujos. Estos flujos realizan desplazamientos cotidianos supeditados al nexo habitabilidadmovilidad. El objetivo de este trabajo consiste en analizar las características de la movilidad urbana relacionadas con el intercambio y transferencia de usuarios en la central nueva de autobuses de Guadalajara, México. Para ello, se utilizaron una serie de instrumentos cuali-cuantitativos auxiliados por software de diseño. En este sentido, la experiencia etnográfica en el sitio recopiló información interesante sobre los procesos de intercambio y transferencia de sujetos. Los resultados muestran que la integración del transporte público y la planificación urbana puede verse desde dos ángulos distintos, aunque relacionados entre sí: a) desarrollo de estrategias y b) elaboración del proyecto. Asimismo, características como la distancia, el tiempo del desplazamiento, la espera y flexibilidad de medios de transporte, son algunas de las posibilidades que han favorecido la aceptación de nuevas modalidades en los desplazamientos territoriales y el uso y adquisición de vehículos particulares. De ahí, la necesidad de brindar soluciones a las necesidades sociales, a través de cambios en el modelo de movilidad.
\end{abstract}

\section{Abstract}

Urban systems are structured through a complex induced network of communication channels. These access roads generally have obstructions and collapses depending on the saturation of the mode used in the transport of living matter and energy. In addition, in certain nodes of the system, there are connectivity and flow transfer processes. These flows make daily trips subject to the habitability-mobility nexus. The objective of this work is to analyze the characteristics of urban mobility related to the exchange and transfer of users in the new bus station in Guadalajara, Mexico. For this, the design software helped to use a series of qualitative and quantitative instruments. In this sense, the ethnographic experience on the site collected interesting information about the processes of exchange and transfer of users. The results show that the integration of public transport and urban planning can be seen from two different, although related, angles, a) strategy development and b) project development. Likewise, characteristics such as distance, travel time, waiting and flexibility of means of transport are some of the possibilities that have favored the acceptance of new modalities in territorial displacements and the use and acquisition of private vehicles. Hence the need to provide solutions to social needs, through changes in the mobility model. 


\section{Introducción}

El espacio público es donde se desarrolla la mayor parte de las dinámicas de movilidad y desplazamiento urbano. Aquí, cualquier persona tendría derecho a circular libremente como flujos de información; su rol es fundamental en el desarrollo y consolidación democrática, fomenta la cohesión e inclusión social, fortalece vínculos interpersonales, participación ciudadana, rendición de cuentas y transparencia administrativa por parte del Estado. No obstante, en este espacio, los sistemas de transporte y las infraestructuras complican o facilitan el ejercicio de la movilidad cotidiana.

Desde una perspectiva termodinámica, las ciudades contemporáneas representan sistemas abiertos que consumen y expiden materia y energía. Son sistemas que experimentan transformaciones morfólogicas de origen multicausal, sujetos a un acelerado desorden sistémico (entropía). Además, tienen fuerte relación con las dinámicas económicas depredadoras y promotoras del consumo irracional de suelo. Concretamente, suelo ubicado fuera de los límites periurbanos; allá donde el vínculo habitabilidad-movilidad evidencia los costos sociales de la construcción horizontal. En suma, las ciudades actuales son sistemas entrópicos con procesos que se alimentan continuamente de recursos provenientes de los sistemas no urbanizados: el ambiente.

En este contexto, las ciudades, entonces, pueden ser concebidas como sistemas. Estos sistemas tienen límites que los diferencian de su ambiente inmediato. Algunos límites suelen ser difusos y cambian de posición en función de la expansión urbano-habitacional. Los límites representan una franja endeble y flexible rodeada de otros sistemas no urbanos ricos en materia y energía que permiten, a costa de su propia homeostasis, el funcionamiento y operación de los procesos urbanos. De esta manera, es posible extraer tres conceptos interrelacionados: "el sistema, la frontera y el ambiente", o en términos urbanos: "el urbano, el periurbano y el no urbano". El primero representaría al sistema de interés o la ciudad. El periurbano, es el límite entre lo que es urbano y lo que no es urbano, y el no urbano, circunscribe los sistemas de soporte; es decir, los sistemas que proveen los insumos necesarios para el funcionamiento del sistema urbano presionante. Asimismo, estos sistemas no urbanos fungen como receptores de la materia y energía no utilizadas (entropía) (González, 2018).

Efectivamente, el urbano ha sido el sistema resultante más impresionante de la presencia antrópica. Es considerado motor de la economía, sinónimo de desarrollo, símbolo de la modernidad y principal productor de oportunidades.
Sin embargo, la entropía en la movilidad y el acceso a los servicios básicos como el transporte ha sido un tema relacionado con descuidos en la cobertura socioterritorial (Gutiérrez, 2010). De ahí, hoy en día las ciudades se han vuelto menos caminables, mayormente fragmentadas, socialmente excluyentes, físicamente dispersas y obscenamente dependientes de la automovilidad particular. Santos, Behrendt \& Teytelboym (2010) comentan que el crecimiento vehicular, la congestión del tráfico y los precios de los combustibles han motivado al estudio de políticas tendientes a racionalizar el consumo del transporte motorizado. Sobre todo, porque "la movilidad resulta un buen reflejo de la dinámica territorial, ya que una de las principales necesidades en las áreas urbanas es el traslado de las personas" (García Schilardi, 2017, p.31).

En América Latina, la movilidad en transporte público representa el principal medio de desplazamiento cotidiano; so pena, del evidente incremento de la motorización privada (Covarrubias, 2013). En este sentido, mientras el desplazamiento implica a una gran cantidad de nodos, vínculos y flujos asociados a la movilidad, y depende de los motivos de viaje, el modo y los medios de transporte, las infraestructuras, conexiones y transferencias, e incide directa e indirectamente en la calidad de vida de los usuarios, la operatividad del servicio, los tiempos de espera y la respectiva concatenación de viajes efectuados en la metrópoli. La movilidad, por su parte, cualquiera que sea su condición y característica, genera una serie de conectividades articuladas y obligadas a la transferencia de los principales sujetos de la movilidad: los usuarios. No obstante, "para una parte de la población, la movilidad se convierte, también, en un factor de desigualdad, pues los desplazamientos se realizan en diferentes condiciones de calidad y tiempo" (Acosta \& Covarrubias, 2018, p.4).

En la década de los noventa son identificadas transformaciones en las políticas de ordenamiento de diferentes sistemas urbanos en países latinoamericanos. Desde entonces, la movilidad se convierte en tema central de diversos estudios de las ciencias sociales, al incorporar nuevas formas de entendimiento (Hasz \& Nigri, 2018). Estas formas de comprensión “(...) supone una capacidad de impactar la garantía del derecho a la ciudad, a la calidad de vida y a un medio ambiente saludable" (Ardila \& Villamizar, 2018, p.21). En este sentido, los procesos de metropolización han configurado una serie de estructuras periféricas urbanas discontinuas e inconexas (entrópicas), con los centros de población, lo cual forma un modelo policéntrico que interactúa en distintas dinámicas urbanas, periurbanas y no urbanas. Este hecho coincide con cifras del Banco de Desarrollo de América Latina (CAF) (2010), debido 
a que en el observatorio de movilidad de este organismo se considera que el futuro de América Latina es urbano, dado que el $80 \%$ de los habitantes de la región, habita en centros de población urbana y la tendencia aumentará hasta el $90 \%$ en los próximos años.

Sumado a lo anterior, según datos de la Comisión Económica para América Latina y el Caribe (CEPAL) cerca de sesenta ciudades cuentan con más de un millón de habitantes, veintitrés con más de dos millones y cuatro ciudades con más de diez millones de habitantes (Rozas, Bonifaz \& Guerra, 2012). En el caso de México, por ejemplo, se contaba hasta el 2010 con 384 ciudades de quince mil habitantes o más, consideradas dentro del sistema urbano nacional y clasificadas en zonas metropolitanas, conurbaciones y centros urbanos, identificados y definidos a partir del marco geoestadístico del Censo Nacional de Población y Vivienda 2010 (SEDESOL-CONAPO-INEGI, 2012). De aquí, la metrópoli de Guadalajara por su parte, es el segundo sistema urbano más importante de México, en cuanto a magnitud poblacional. Es decir, pasó de ser una zona metropolitana constituida por los municipios de Zapopan, Tlaquepaque y Tonalá (ZMG), a un Área Metropolitana de Guadalajara (AMG) conformada por los municipios antes mencionados más los municipios de El Salto, Juanacatlán, Tlajomulco de Zúñiga, Ixtlahuacán de los membrillos y Zapotlanejo.

En agosto del 2013 es cuando empiezan a ocurrir cambios importantes en materia de movilidad. Se sustituyeron leyes que pretendían ordenar e integrar las distintas modalidades de transporte, a través de la puesta en marcha de la nueva Ley de Movilidad y Transporte para el estado de Jalisco; se creó el Instituto de Movilidad (hoy extinto), el Registro Estatal de Movilidad, en reemplazo del Organismo Coordinador de la Operación Integral del Transporte Público (OCOIT) y el Centro Estatal de Investigación de la Vialidad y el Transporte (CEIT). Asimismo, la Secretaría de Vialidad pasó a ser la Secretaría de Movilidad (actualmente Secretaría de Transporte) y se inician las obras de construcción de la tercera línea del tren ligero (actualmente existen dos líneas en operación: línea 1 norte-sur y línea 2 de oriente-poniente). Sobre esta última infraestructura (línea 3), las características son similares a las de un tranvía regular; sin embargo, opera en plataforma segregada del resto del tráfico, con derecho de paso prioritario en los puntos de intersección. Tienen una capacidad intermedia de transporte a escala regional y metropolitana, generalmente mayor que la del tranvía y menor que la del ferrocarril convencional. El proyecto consta de 21.5 kilómetros, con trece estaciones elevadas y cinco estaciones subterráneas. Recorre diagonalmente la metrópoli (suroriente a norponiente) y conecta a tres municipios: Zapopan, Guadalajara y Tlaquepaque.

En cuanto al autotransporte público (buses), fue propuesta una reestructuración en los recorridos de las rutas y el cambio en el esquema de trabajo de los prestadores de este servicio. Es decir, una transición del modelo hombrecamión (hombre-autobús) a un modelo denominado ruta-empresa con la finalidad de integrar los diferentes sistemas de transporte disponibles en la metrópoli. En este sentido, las acciones presuponen una homologación de los salarios de los operadores y el cobro de tarifas (peajes) por medio del prepago del pasaje, a través de tarjetas inteligentes. Igualmente, se promovió incentivar la movilidad no motorizada e incrementar el consumo de transporte público, el equipamiento e infraestructuras como andadores temáticos, calles de preferencia ciclista, el woonerf (patio viviente) holandés, las zonas de accesibilidad preferencial o zonas 30 del tipo holandés y alemán; donde la velocidad permitida es de $30 \mathrm{~km} / \mathrm{h}$, la conceptualización y divulgación del traffic calming, entre otras acciones que promueven el paradigma de la denominada Movilidad Urbana Sostenible (MUS).

En función de lo anterior, esta investigación cuestiona la integración de conectividades y transferencia de usuarios en la estructura urbana de la metrópoli de Guadalajara. Concretamente, en la denominada central nueva de autobuses, uno de los principales nodos de conectividad y transferencia dentro de la metrópoli. Efectivamente, la movilidad tiende a restringirse o reducirse a nivel local, pero llega a afectar a más del 70\% de la población nacional (ITDP, 2013). De esta forma, el objetivo de este trabajo consiste en analizar las características de la movilidad urbana en este nodo de intercambio y transferencia de usuarios (figura 1).

La central nueva de autobuses se inauguró en abril de 1988, en el intento de remplazar a la central de autobuses "Los Ángeles" o central vieja. Este nodo lleva por nombre formal central de autobuses Salvador Sánchez Alcántara y representa uno de los más importantes centros de conectividad y transferencia del país. El proyecto fue planteado como una alternativa de solución para resolver algunos problemas de movilidad motorizada que ya experimentaba la ciudad en esa época, principalmente con el ingreso de autotransportes a la zona céntrica del barrio tradicional de Analco. Se edificó en un periodo aproximado de dos años, el cual propició la especulación inmobiliaria del entorno, se construyeron centros comerciales anexos, vivienda de interés social y las existentes colonias populares detonaron económicamente con servicios y productos de consumo para la industria del autotransporte. Fue inaugurada 


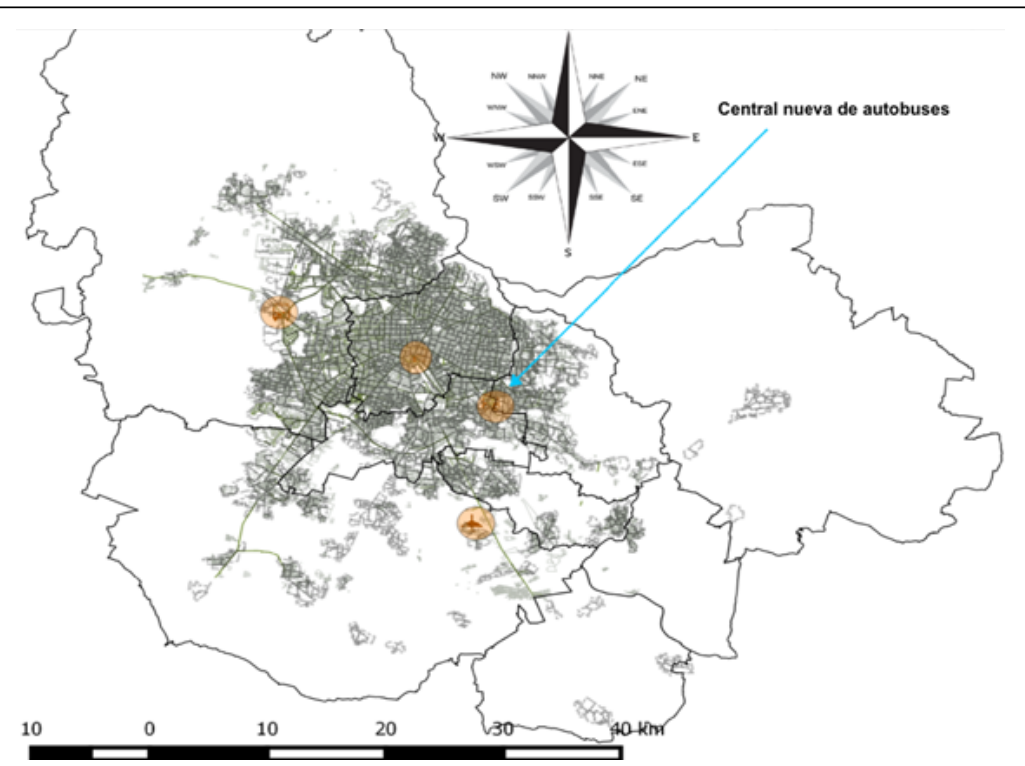

Figura 1. Ubicación de la central nueva de autobuses en la metrópoli de Guadalajara. Fuente: Elaboración propia (2019).

en 1988, desplantando el inmueble en las confluencias de la autopista a México y la carretera libre Zapotlanejo con la visión de seguir manteniéndose a la vanguardia, considerada así desde su concepción por los grupos de autotransportistas, hoy integrados a la CANAPAT, como la más grande y funcional del país, iniciando operaciones en esta terminal con viajes al pacífico, el norte y noroeste del país (El Informador, 1988).

En este contexto, a tres décadas de su puesta en marcha, ha dispersado sus servicios al igual que la ciudad su crecimiento, donde se han diversificado en pequeñas terminales adyacentes al inmueble y oficinas de servicio en los perímetros y puntos clave en la metrópoli. En marzo de 2016, el nodo contenía siete módulos con veintisiete líneas de transporte foráneo que brindaban el servicio de pasajeros. De igual forma, conectaba a los usuarios de la metrópoli por medio de autobuses intraurbanos e interurbanos, autos de alquiler y, próximamente, la tercera línea de tren ligero. La oferta del servicio para el desplazamiento interurbano lo complementan autobuses de trece empresas de transporte colectivo urbano y suburbano, dos organismos descentralizados del gobierno estatal, automóviles de alquiler exclusivo del sitio de taxis de la central de autobuses, taxis de otros sitios que no ingresan al área de módulos, automóviles contratados por medio de aplicaciones móviles; generalmente de Uber, vehículos particulares y alternativas clandestinas surgidas en la periurbanización como respuesta a la falta de autotransporte público intracondominal conocidos popularmente como mototaxis (figura 2 y figura 3 ).

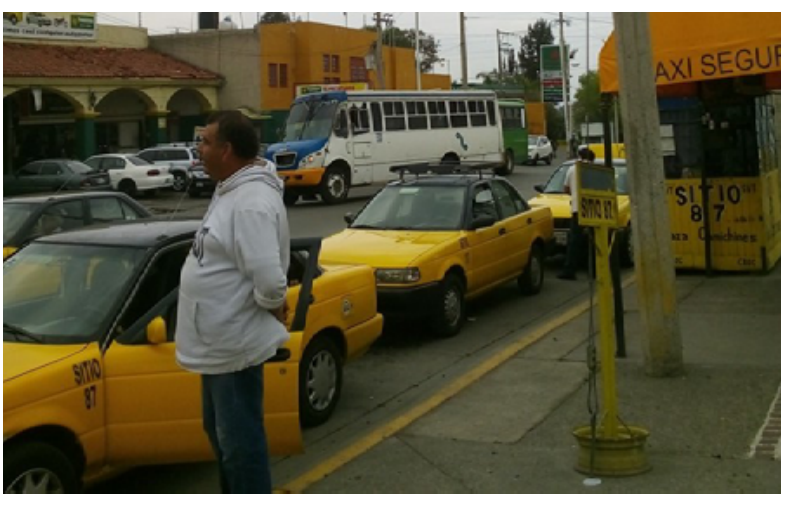

Figura 2. Sitio de taxis. Fuente: Elaboración propia (2019).

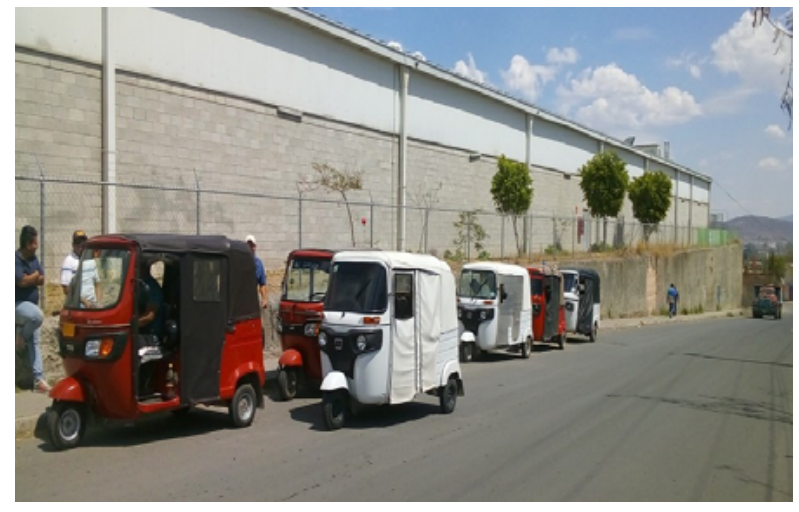

Figura 3. Sitio de mototaxis. Fuente: Elaboración propia (2019). 


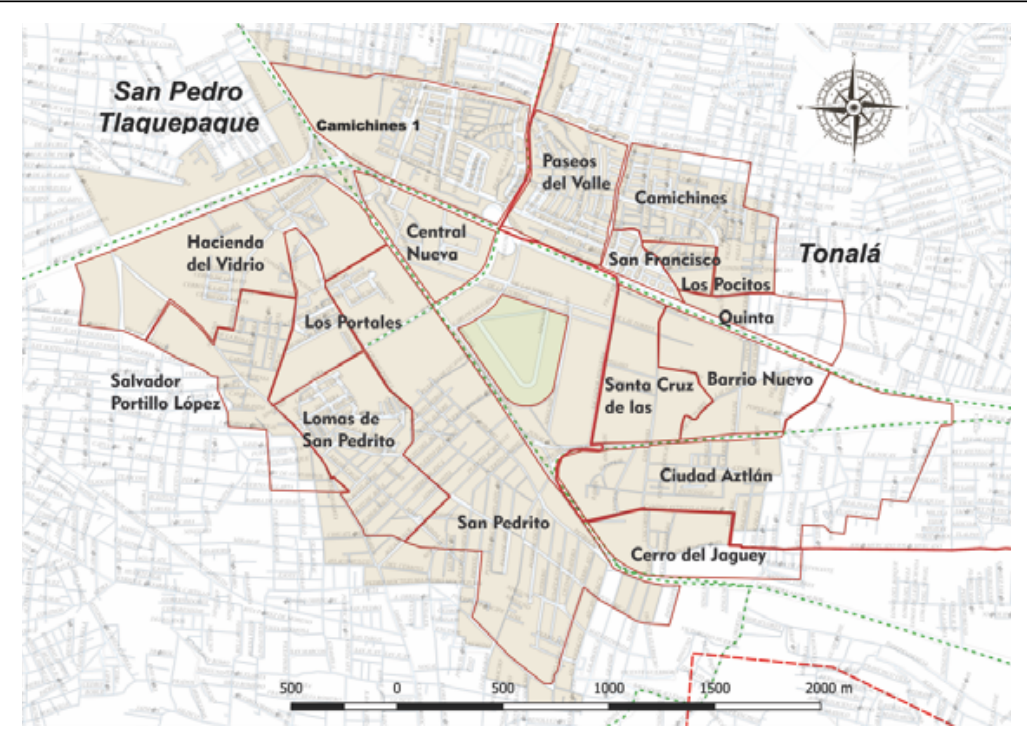

Figura 4. Delimitación de las colonias vecinas a la central nueva de autobuses. Fuente: Elaboración propia (2019).

La central nueva de autobuses es el nodo con mayor conectividad, intercambio, transferencia de diferentes modos de transporte terrestre y concatena la movilidad urbana motorizada y no motorizada, con una gran cantidad de transportes ubicados en la conurbación municipal de Tonalá y San Pedro Tlaquepaque. El primer municipio, según datos de SEDESOL-CONAPO (2012), cuenta con un territorio de $119.6 \mathrm{~km}^{2}$ y una población de 536.111 habitantes. De aquí, 33.356 están distribuidos en nueve colonias vecinas a la central nueva de autobuses (Paseos del Valle, San Francisco, Camichines Sección II, Santa Cruz de las Huertas, Barrio Nuevo, Ciudad Aztlán, Los Pocitos, Quinta Catalina y Colinas de la Cruz). En el caso del municipio de San Pedro Talquepaque o Tlaquepaque, su extensión territorial es de $270.88 \mathrm{~km}^{2}$ y población de 664.193 habitantes. De estos, 35.349 radican en nueve colonias contiguas: Crucero central, Camichines Sección I, Cerro del Jagüey, San Pedrito, Lomas de San Pedrito, Salvador Portillo López, Los Portales, Hacienda de Vidrio y Central Nueva.

"El acceso a la ciudad y a sus diferentes servicios está determinado en parte por la infraestructura del transporte, y esta, a su vez, por la estructura urbana de la ciudad" (Hernández, 2018, p. 129). En este sentido, la morfología urbana y las dinámicas sociales con que se realizan los intercambios y conectividades han ido desarrollando numerosas soluciones a conflictos adyacentes que influyen en el desarrollo económico, social, medioambiental y principalmente en la calidad de vida y salud de los habitantes. De tal forma, es posible observar rutas de autotransporte público (urbanas) que circulan en carreteras $\mathrm{y}$ transportes suburbanos que operan en el interior de la ciudad, es decir, urbaneando. Este término popular en los operadores del autotransporte público local hace referencia al proceso de ejercer movilidad motorizada en la urbe. En este sentido, las carreteras, calles y avenidas terminan convirtiéndose en simples ingresos a zonas habitacionales y los vehículos particulares o bien de servicio público en habitáculos resultado de las horas que se invierten en realizar los desplazamientos cotidianos. Por ello, el reglamento estatal de zonificación para el estado de Jalisco, a través del sistema de unidades urbanas pretende el ordenamiento del espacio urbano en centros de población. De aquí, las colonias adyacentes al inmueble de la central nueva de autobuses se asientan en un radio de influencia, no mayor a dos kilómetros de distancia (Figura 4).

El área de estudio se encuentra acotada por dos ejes carreteros regionales de gran demanda vehicular: a) la autopista de cuota a León No. 80 y b) la carretera libre a Zapotlanejo. No obstante, ambas llegaron a su límite de saturación (SCT, 2012). Ello debido a que los tramos que conducen a Tonalá, El Salto y Tlaquepaque han incrementado la entropía causada por la circulación y los desarrollos habitacionales adyacentes. Además, desde el 2017 se han presentado cierres viales provenientes de las obras de construcción de la línea 3 del tren ligero y accidentalidad. Estos accidentes, hechos o percances viales han provocado preocupación en las autoridades encargadas de planificar la ciudad; particularmente, las que revisan la entropía de la movilidad y el transporte. 


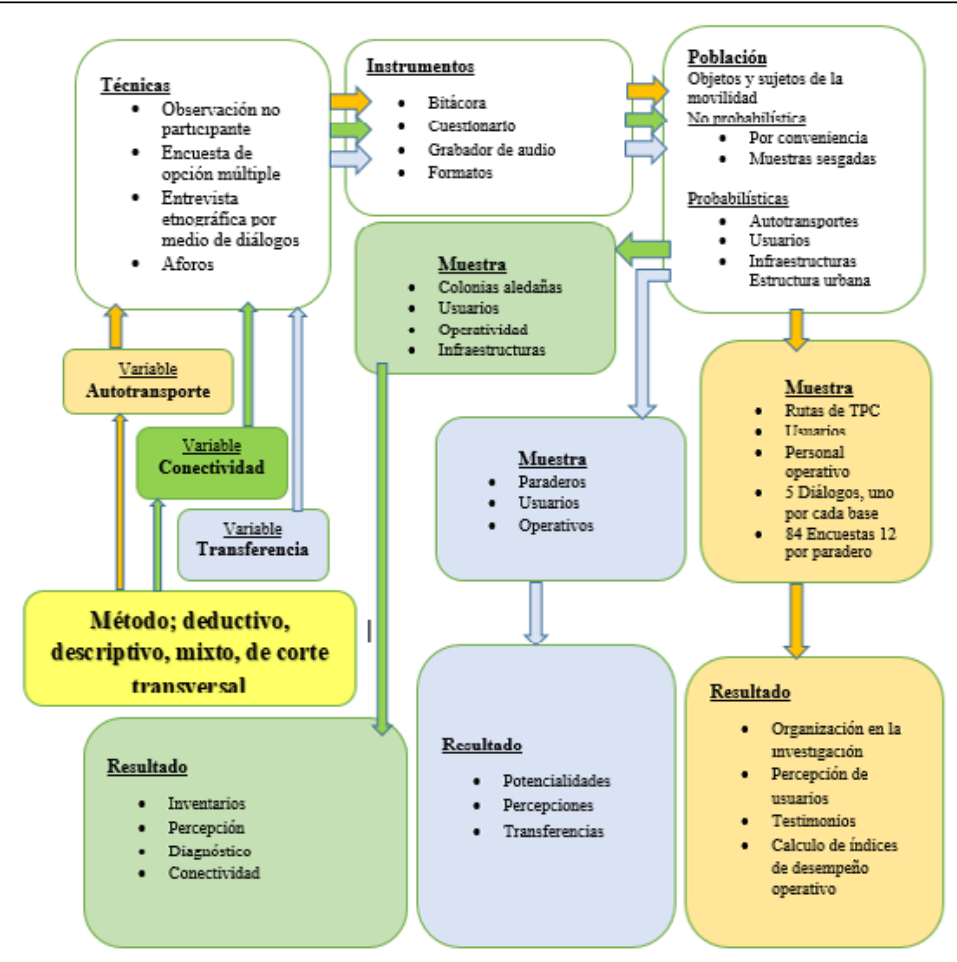

Figura 5. Resumen metodológico. Fuente: Elaboración propia (2019).

Aquí, hay coincidencia en torno a que "(...) la ocurrencia de este tipo de eventos se aleja de factores puramente estocásticos, que son resultado de anomalías asociadas con la interacción intrasistémica del vehículo, el usuario y el camino" (García, González \& Asprilla, 2018, p. 51). Por otro lado, la antigua carretera a Tonalá es otra vialidad con menor demanda de tránsito vehicular, respecto a la No. 80 y la libre a Zapotlanejo; sin embargo, su tránsito es complicado para peatones, ciclistas o motociclistas debido al giro comercial adyacente al corredor vial, por el incremento en comercios y usos de suelo mixto que la convierten en una vía de riesgo para el peatón, por la circulación de vehículos de carga pesada, autotransporte público, vehículos particulares, vehículos adaptados para pasajeros y otros.

\section{Materiales y métodos}

En un primer momento el presente estudio realizó una indagación documental a través de la recopilación de información referente al transporte, la estructura urbana y las infraestructuras para la movilidad. Se describieron la interacción entre sujetos y objetos, nodos y transferencia. Efectivamente, se vuelve complejo generalizar e identificar isomorfismos, es decir, homogeneidad de características. Sobre todo, cuando intervienen diversas variables, sin embargo, es posible "(...) intentar generalizar una metodología aplicable a todos los casos particulares" (Díaz \& De Ureña, 2010). De esta forma, si se consideran estadísticas oficiales y observación de campo, se obtuvo información del área de estudio: sus características físicas, equipamiento, sistemas de desplazamiento y reparto modal. Para ello, se realizó la construcción de un diagnóstico, donde fue necesario verificar y realizar una inspección in situ y describir las características socioterritoriales, usos y destinos del suelo que existen en la estructura urbana, e identificar los espacios de encuentro y convivencia social. En un segundo momento, fue obtenida la cantidad de pasajeros que arriban a cada punto de ascenso y descenso, se determinó la ubicación, distancia y destino. Posteriormente, estos datos se fueron contrastando con diálogos abiertos de operadores de transporte colectivos e intracondominales (etnografía). Se aplicaron ochenta y cuatro encuestas a usuarios y residentes de zonas de proximidad con la intención de describir la percepción, en cuanto a operatividad y seguridad en la estructura urbana (figura 5).

Las encuestas fueron realizadas por conveniencia. Es decir, se realizó un muestreo no probabilístico, tomando elementos representativos, para obtener datos precisos sobre la percepción y operatividad de los sistemas de transporte. 


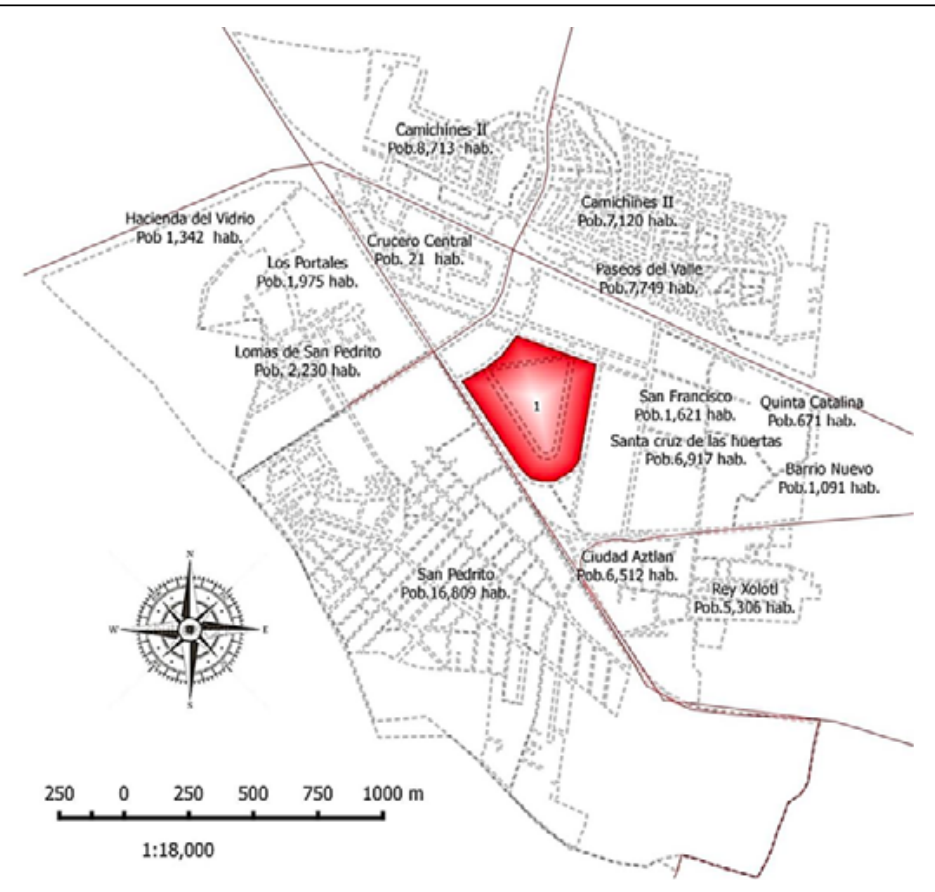

Figura 6. Polígono de influencia de la nueva central de autobuses de Guadalajara. Fuente: Elaboración propia con base a datos 2015 del IIEG Jalisco, 2019.

Los criterios cualitativos de selección se integraron en unidades de análisis por medio de cuotas. Además, se requirió establecer un diálogo sin restricciones a través de un cuestionario con interrogantes precisas vinculadas a la operación e integración de conectividades y transferencias. En resumen, fue preciso obtener la experiencia. De ahí, fueron elaborados cinco diálogos abiertos con diferentes actores sociales (usuarios, taxistas, choferes de autobús, choferes de empresas de redes de transporte) en paraderos y nodos intercambio de pasajeros.

Esta población objetivo tenía la cualidad isomorfa de desempeñar su función laboral en las inmediaciones a la central nueva de autobuses. Fue necesario también: a) Observar pasajeros que abordaban y descendían en cada parada de autobús, es decir, en los puntos de concentración y transferencia de usuarios; b) Identificar divergencias, convergencias y potencialidades de los intranodos urbanos, concretamente el referente a la central nueva de autobuses; c) Vaciar la información mediante el apoyo del software de georeferenciación Qgis y Autocad para la elaboración de mapas y definir la información obtenida y d) Calcular el índice de Pasajeros por Kilómetro (IPK), Índice de pasajeros por vehículo (IPV) y Vehículos por kilómetro (VK). El polígono de estudio se circunscribe en un radio de influencia no menor a 800 metros (figura 6). Esta superficie contiene infraestructuras para la movilidad, puentes peatonales, banquetas, senderos, semaforización, paraderos, autos de Uber, taxis y mototaxis. De tal forma que fue posible realizar inventarios de equipamiento e infraestructuras, así como aforos de ocupación de transporte.

\section{Resultados}

El polígono territorial influenciado por la central nueva de autobuses ha consolidado un nodo clave para la conectividad y transferencia de usuarios del transporte público, contiene dieciocho colonias asentadas en sus entornos, con una mezcla de uso de suelo mixto y su respectiva relación económica que hace evidente el intercambio comercial. Además, circunscribe un radio de influencia no mayor a $2 \mathrm{~km}$ de distancia, esto le permite desplazamientos no motorizados, dotación de equipamiento jerarquizado y dosificado de infraestructuras.

El nodo de la central de autobuses es servido por veintisiete rutas de transporte público, 780 taxis asignados al servicio exclusivo de la central (figura 7). Sin embargo, en sus alrededores se encuentran bases de estos servicios y medios alternativos como mototaxis y autos de Uber. En el caso de estos últimos, la información que se maneja no es tan accesible y los mototaxis no cuentan con una estadística oficial. De ahí, la información vertida provino de los comentarios de socios, conductores y usuarios. 


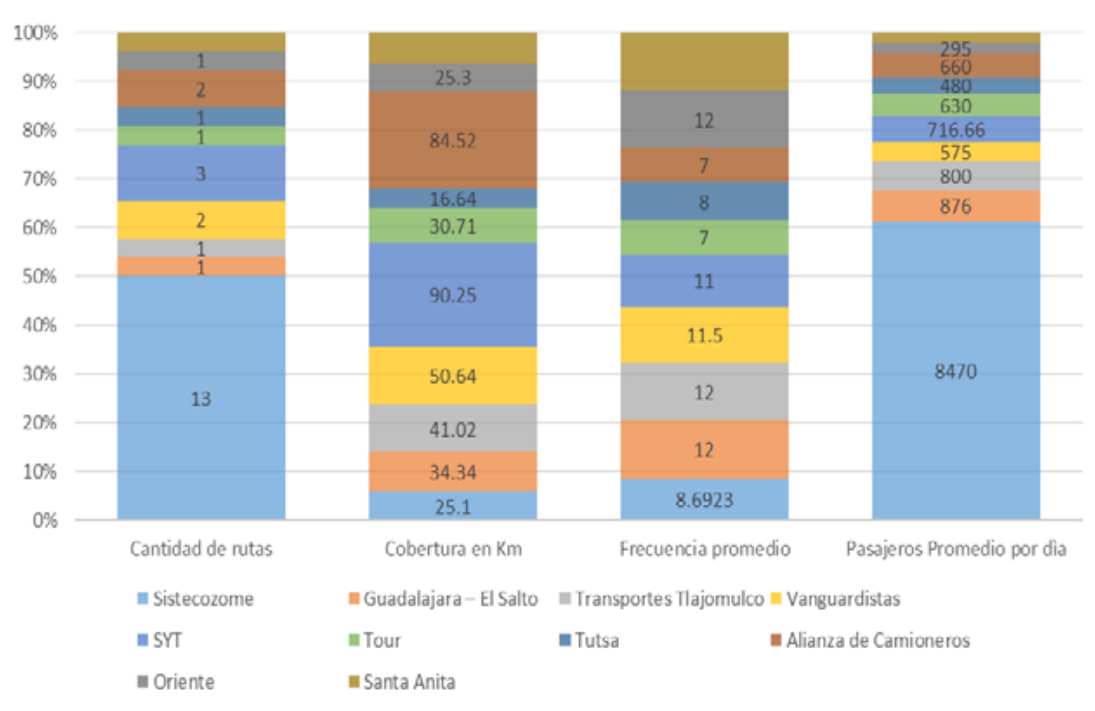

Figura 7. Rutas de transporte público en la central nueva de autobuses. Fuente: Elaboración propia (2019).

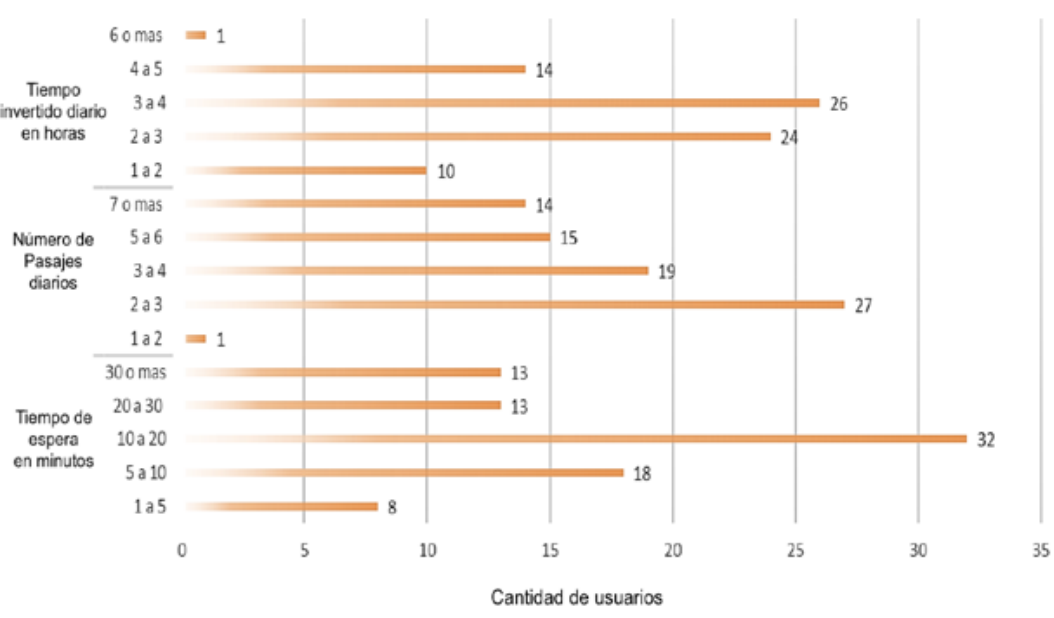

Figura 8. Reparto modal de la movilidad cotidiana de los usuarios entrevistados. Fuente: Elaboración propia (2019).

Se obtuvieron datos importantes sobre accesibilidad de los usuarios mediante la ubicación de paraderos. Esto facilitó la información sobre la conectividad territorial que tienen los sistemas de autotransporte al interior de la metrópoli. En consecuencia, la integración del transporte público y la planificación urbana puede verse desde dos ángulos distintos, aunque relacionados entre sí: a) desarrollo de estrategias y b) elaboración del proyecto. En este sentido, la encuesta reflejó la preferencia o posibilidad del usuario sobre el medio de desplazamiento en el área de estudio (figura 8).
Otros rasgos característicos como la distancia, el tiempo de desplazamiento, tiempos de espera y flexibilidad de medios de transporte son algunas de las posibilidades que ha favorecido la aceptación de nuevas modalidades en los desplazamientos territoriales y el uso o adquisición de vehículos particulares (figura 9). Aquí, se vuelve prioritario determinar soluciones sociales a través de cambios en el modelo de movilidad, es decir, la construcción de una relación entre infraestructuras, medios y modos.

Por otro lado, el índice de pasajero por kilómetro (IPK) es uno de los más conocidos. Este relaciona la efectividad 


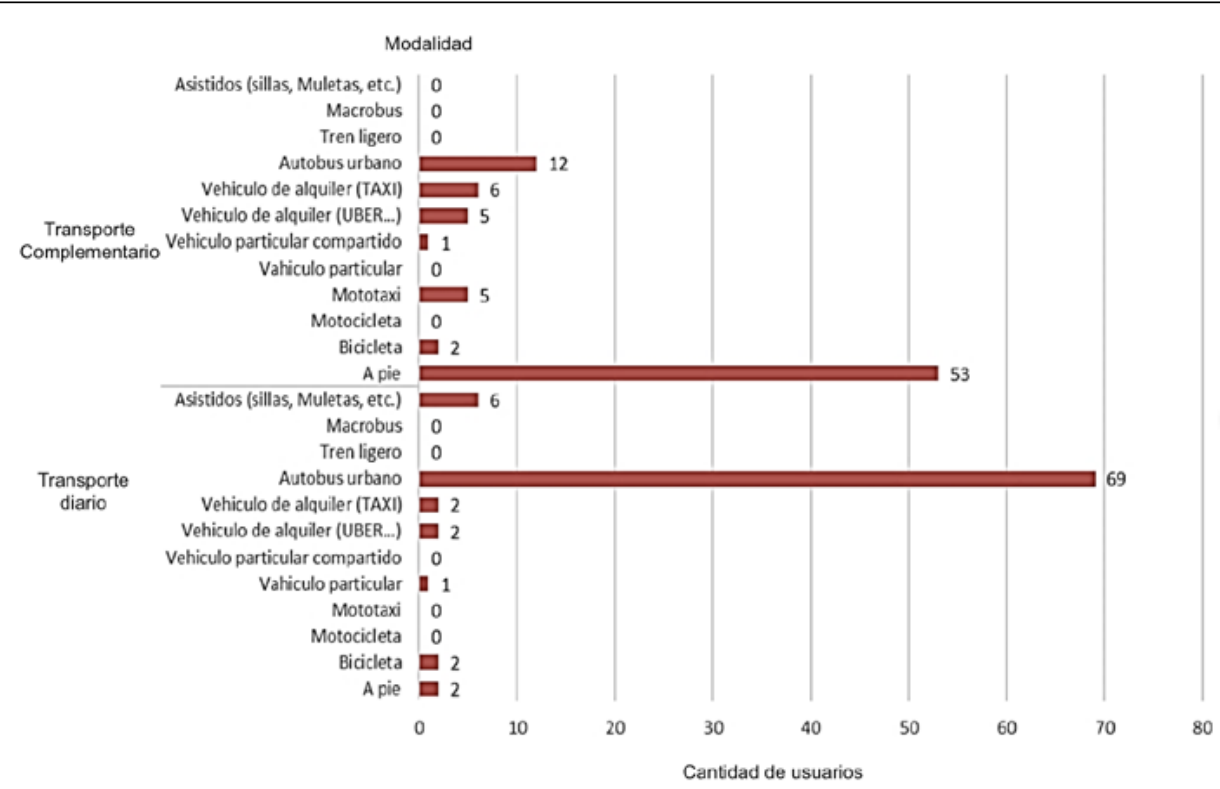

Figura 9. Tiempo e inversión en el transporte de los usuarios entrevistados. Fuente: Elaboración propia (2019).

y eficiencia del sistema de transporte con el resultado (Pasajeros) y un producto (Kilómetros). Se obtiene dividiendo el número de pasajeros por kilómetro recorrido. El índice de pasajeros por vehículo (IPV), por su parte, identifica simultáneamente la operatividad, capacidad del sistema y nivel de servicio, lo que arroja como resultado la densidad de pasajeros en el transporte. El volumen de vehículos por kilómetro (VK) permite dar seguimiento a la oferta del sistema de transporte colectivo, en un comparativo entre rutas de transporte. Por lo regular el VK debe ser evaluado en conjunto con otros indicadores de transporte si existiera la infraestructura y compararse con la oferta real de los otros sistemas de transporte (Tabla 1).

La percepción de los usuarios del transporte en el área de estudio muestra que la mayoría lo califica de mala calidad o pésima. Esto es muy posible, debido a que no se encuentra integrado o no proporciona la seguridad en el entorno de los paraderos y se constata con el incremento de los medios motorizados de transporte privado (figura 10).

Las cifras y los índices de motorización demuestran que los medios del desplazamiento particular se incrementan no solo en los ingresos carreteros, como muestran los datos de los aforos en estaciones permanentes de la SCT (2012), en los dos ejes carreteros que delimitan el área de estudio, lo que mantiene una hegemonía sobre los transportes colectivos o masivos, sino que el incremento proporcional que guardan dichos elementos determinantes de la movilidad urbana. En los últimos cinco años, la
Tabla 1

IPK, IPV y VK de las rutas de transporte en la central nueva de autobuses.

\begin{tabular}{lccc} 
Ruta & IPK & IPV & VK \\
\hline 610 & 25.18 & 14.36 & 1.75 \\
$610 \mathrm{~A}$ & 31.33 & 42.78 & 0.73 \\
611 & 40.40 & 11.19 & 3.61 \\
611 A & 44.41 & 11.83 & 3.76 \\
616 & 33.32 & 22.75 & 1.46 \\
619 & 31.09 & 5.77 & 5.38 \\
619 San Agustín & 18.49 & 30.86 & 0.60 \\
643 & 31.45 & 16.35 & 1.92 \\
643 Urbi quinta & 22.04 & 29.41 & 0.75 \\
644 B Carril & 37.43 & 48.50 & 0.77 \\
644 B Jauja & 35.56 & 57.25 & 0.62 \\
644 B San Martín & 24.12 & 41.25 & 0.58 \\
644 B Santibáñez & 26.18 & 51.92 & 0.50 \\
178 El Salto & 18.84 & 30.81 & 0.61 \\
187 Tlajomulco & 17.58 & 27.73 & 0.63 \\
207 & 20.43 & 20.00 & 1.02 \\
59 & 23.07 & 19.56 & 1.18 \\
275 & 15.77 & 20.50 & 0.77 \\
275 A & 25.50 & 32.45 & 0.79 \\
275 SB & 18.61 & 57.70 & 0.32 \\
706 & 19.08 & 21.70 & 0.88 \\
330 & 45.01 & 32.57 & 1.38 \\
380 & 12.55 & 5.73 & 2.19 \\
80 A & 35.07 & 36.76 & 0.95
\end{tabular}

Fuente: Elaboración propia. 


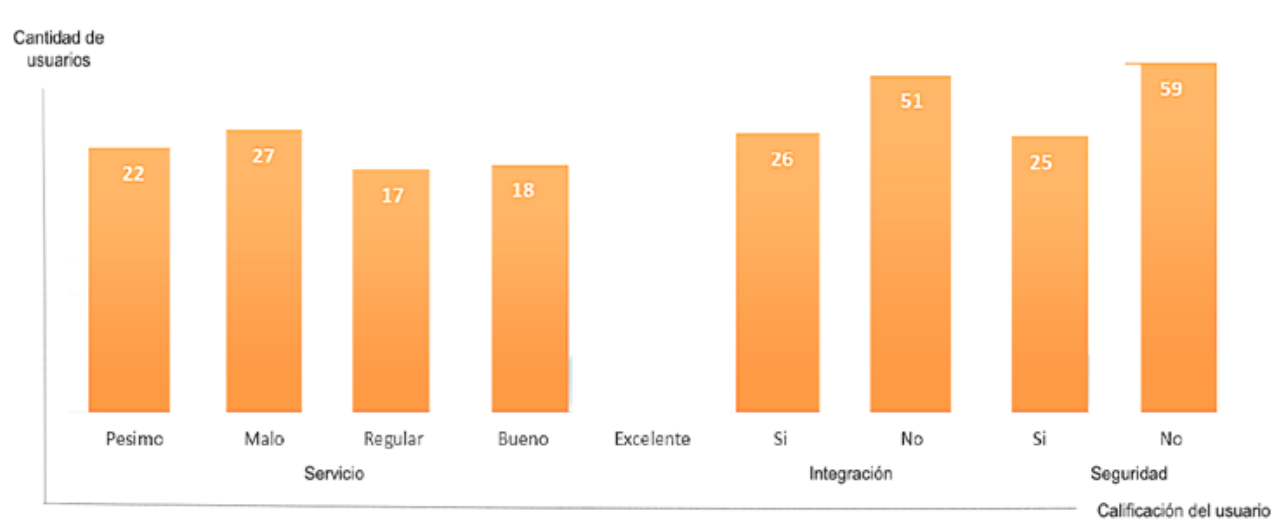

Figura 10. Percepción de la calidad en el servicio en la central nueva de autobuses. Fuente: Elaboración propia (2019).

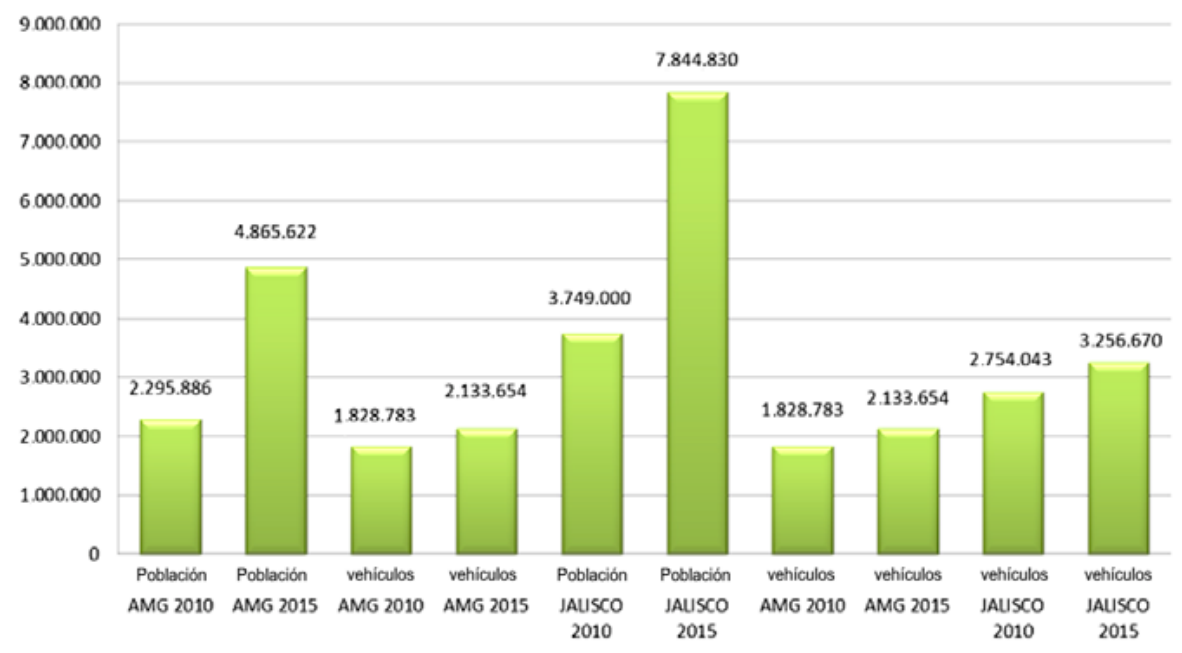

Figura 11. Incremento poblacional y motorización. Fuente: Elaboración propia con datos del IIEG (2015).

metrópoli muestra un incremento de población y vehículos registrados en $3.90 \%$ con respecto a 2010 (figura 11).

\section{Discusión}

"En la actualidad, las infraestructuras de transporte son construidas y reconstruidas en función del paradigma en términos kuhnianos de la movilidad motorizada" (Asprilla, González \& García, 2018, p. 626). Aquí, la preponderancia del automóvil supone la ocupación creciente de espacio público para circulación y aparcamiento. No obstante, la cantidad y capacidad de la infraestructura se ve rebasada y afecta los tiempos de traslado, a través de la disminución de la velocidad permitida en las horas de mayor demanda
(González, 2018. De ahí, la urgencia de reducir el consumo de los medios de transporte motorizados y replantear el diseño de espacios urbanos que beneficien al peatón. Ciertamente, ONU-Hábitat no ha tenido recursos financieros para proyectos a largo plazo relacionados con el apoyo a la planificación urbana en cualquiera de las ciudades. Así, mientras ha trabajado para asegurar fondos adicionales de otros donantes o programas para continuar el trabajo, en cambio, diferentes donantes financiaron actividades y productos tales como: el plan espacial para Meuraxa Kecamatan, o el Plan de Recuperación y Reconstrucción de Tacloban (Maynard, Parker, YosephPaulus \& García, 2018). 
En este contexto, "los procesos futuros de expansión urbana en las ciudades latinoamericanas han sido poco estudiados a pesar de que el bienestar de sus ciudadanos dependerá de la gestión territorial y de la planificación de la provisión de beneficios y servicios ecosistémicos" (Bonilla, Mora, Vaca, Estrella \& Herrera, 2020, p. 1). Efectivamente, las dinámicas urbanas de movilidad configuran modelos de producción y organización del territorio como resultado del reajuste de las actividades económicas. Sin embargo, un cambio de paradigma se vuelve impostergable, no solo en la movilidad y el transporte, sino a escala territorial. Ello porque al cambiar la infraestructura, infaliblemente cambia la sociedad en su conjunto, las relaciones de poder, las instituciones y los elementos de la superestructura, transformando los patrones de desplazamiento, el medio y modo de transportarse estratégicamente para favorecer la circulación dentro de la metrópoli. Aunque este no se limita solamente a la construcción de infraestructuras, sino que se requieren esfuerzos de gestión e innovación en materia de financiamiento.

La composición de la accesibilidad y conectividad de los nodos de transporte combinados con la mixtura de los usos de suelo y sus proximidades con servicios, comercio, vivienda, espacios de recreación y contenidos juegan un papel preponderante para consolidar el paradigma de la movilidad urbana sostenible. Sin embargo, "la planificación urbana aún carece de estándares apropiados para definir los límites de la ciudad en los sistemas urbanos (Sotomayor \& Samaniego, 2020, p. 1), donde la expansión urbana ha generado impactos negativos a la biodiversidad (Mimet, Kerbiriou, Simon, Julien \& Raymond, 2020). En este sentido, "las relaciones espaciales entre la intensidad del uso del suelo urbano y la vitalidad urbana requieren atención inmediata a fin de presentar apoyo para la toma de decisiones y sugerencias para el futuro desarrollo urbano y planificación" (Xia, Yeh \& Zhang, 2020, p. 1). En función de lo anterior, las vivencias de las identidades móviles en el entorno de la central nueva de autobuses han determinado la percepción individual del espacio o viaje con fuertes dosis de entropía intrasistémica. El radio de influencia de la central nueva de autobuses es una puerta de ingreso-salida de la ciudad por medios terrestres, es un nodo articulador para las movilidades cotidianas interurbanas, cumple funciones de estancia, atracción y detractor intermunicipal de los sujetos de la movilidad. Sin embargo, provoca entropía al segregar población adyacente, quienes pudieron haberse incorporado al proyecto en términos de accesibilidad, conectividad, comercio y turismo religioso, como es el caso de la comunidad de San Martin de la Flores. En este poblado, anualmente se celebra el viacrucis de Semana Santa y es visitada por miles de turistas locales y regionales. Igualmente, las colonias vecinas de Tonalá han quedado desvinculadas, debido a la autopista que delimita al nodo y deja inconexión. Estas características específicas consideran una serie de relaciones existentes entre el espacio interior y las distintas partes que conforman la ciudad, con un estatus público o privado, lo que continúa sucesivamente por zonas centrales y periféricas que se van desarrollando de acuerdo a las directrices de la urbanización y se distribuyen a partir de un núcleo inicial, con un orden determinado por sí mismas, su topografía, usos del suelo o por el mercado inmobiliario.

Al considerar que la ciudad surge a lo largo de la historia como un nodo topológico de intercambio, que va conformando en función del tiempo su estructura y morfología urbana. El trazo de la red viaria, por tanto, puede analizarse de forma radial, ortogonal u orgánica, incluso, encontrarse de forma combinada. Las vialidades son componentes básicos de la ciudad, sin embargo, en la actualidad están frecuentemente colapsadas por la saturación casi exclusiva de los medios de desplazamiento individuales motorizados, aún y cuando parcialmente logran satisfacer necesidades de movilidad, pero terminan induciendo el consumo del vehículo particular con secuelas entrópicas en materia de contaminación y consumo de energías fósiles.

\section{Conclusiones}

La movilidad de las personas en la central nueva de autobuses debe estar por encima de los vehículos y las infraestructuras para estos o, en otros términos, es necesario consolidar modos eficientes de transporte público e impulsar infraestructura para el uso de bicicleta y el peatón. Efectivamente, se le sigue restando valor a medios lentos de desplazamiento, para minimizar la importancia de las estrategias de gestión de la demanda y al impacto de la estructura urbana en la movilidad y accesibilidad de los usuarios. Es preciso señalar que esta investigación ha arrojado datos de una realidad socioeconómica en un territorio en estado de transformación, sobre todo, porque algunas de las mediciones cualitativas y cuantitativas sobre conectividad e integración de los medios de transporte urbano provocan decisiones personales para la selección de algún medio de desplazamiento.

La seguridad y conectividad de las infraestructuras intraurbanas solo han pensado en restructurar el transporte hacia el interior. El actual sistema de transporte público de la metrópoli conserva una serie de deficiencias como la sobreposición de rutas, saturación de vías principales, 
poca o nula cobertura de servicio en zonas habitacionales periurbanas, inadecuadas condiciones del parque vehicular, inoperatividad del transporte desde una concepción sistémica y discreción en la decisión de las tarifas.

En la dimensión urbano-metropolitana surgen alternativas para la movilidad no precisamente para satisfacer fines ténico-ingenieriles, sino para atender necesidades sociales relacionadas con la reducción de entropías de conectividad, tiempos y costos de los trayectos cotidianos. Igualmente, el tema relacionado con el consumo excesivo del vehículo particular en los desplazamientos intraurbanos y las rutas del transporte colectivo, por su parte, se identifican como conductos capilares que contienen una gran cantidad de puntos de ascenso y descenso de pasajeros definidas por la autoridad y otras sin un reconocimiento oficial.

En muchos tramos de las rutas hacía la central nueva de autobuses de la metrópoli de Guadalajara, es posible encontrar sobreposición de rutas (traslape de rutas) que compiten arduamente entre ellas y con otros medios de transporte por el pasaje, sobre todo cuando estos no cubren las necesidades de desplazamiento de la población usuaria. En este sentido, al no encontrar oferta tradicional en los servicios de transporte aparecen medios alternativos que solo vienen a incrementar el desorden sistémico, es decir, la entropía.

En este contexto, la gestión de la movilidad debe ser vista como un conjunto de estrategias direccionadas a cambios en el comportamiento de los viajes y responder a ¿cómo?, ¿cuándo? y ¿dónde viaja la gente?; esto con el fin de incrementar la eficiencia de los sistemas de transporte. De tal manera, es prioritario establecer objetivos específicos para la conformación de políticas públicas enfocadas a impulsar la movilidad urbana sostenible. Dichas estrategias de gestión de la movilidad coinciden con diferentes opiniones que homogéneamente se han enfocado en mover personas y bienes de forma eficiente.

\section{Referencias}

Acosta, S. Alex \& Covarrubias, A. (2018). La gobernanza del transporte público urbano en México: un comparativo de las localidades de Hermosillo, Sonora y León, Guanajuato. Estudios sociales, 18(52), 1-28. https://doi.org/10.24836/es.v28i52.549

Ardila, A. \& Villamizar, N. (2018). Ciudad(anía) en movimiento: construcción social de instrumentos de políticas de movilidad en Bogotá y Belo Horizonte 1995-2015. Universitas Humanística,
85(85), 19-57. https://doi.org/10.11144/Javeriana. uh85.cmcs

Asprilla, Y., González, M. \& García, F. (2018). Entropía en la periurbanización: desigualdad en el acceso a las infraestructuras de transportes en Tonalá, México. urbe. Revista Brasileira de Gestão Urbana, 10(3), 624-636. https://dx.doi. org/10.1590/2175-3369.010.003.ao10

Bonilla, S., Mora, A., Vaca, A., Estrella, A. \& Herrera, M. A. (2020). Modelling the relationship between urban expansion processes and urban forest characteristics: An application to the Metropolitan District of Quito. Computers, Environment and Urban Systems, 79(1). https://doi.org/10.1016/j. compenvurbsys.2019.101420.

CAF. (2010). Banco de desarrollo de América Latina y el Caribe. Observatorio de movilidad urbana para América Latina. Bogotá, Colombia: Corporación Andina de Fomento. Recuperado de https://www.caf. com/media/4203/desarrollourbano_y_movilidad americalatina.pdf

Covarrubias, A. (2013). Motorización tardía y ciudades dispersas en América Latina: definiendo sus contornos, hipotetizando su futuro. Cuadernos de vivienda y urbanismo, 6(11), 12-43.

Díaz, S. E. \& De Ureña, J. M. (2010). El Estudio del papel territorial de los intercambiadores de transporte. Boletín de la Asociación de Geógrafos Españoles, 1 (54), 29-56.

El Informador (15 de abril de 1988). La nueva Central Camionera será abierta el día 30. El informador, p. 263.

García, N. F., González, M. \& Asprilla, Y. (2018). Determinación de los componentes entrópicos de la accidentalidad: el trinomio vehículo/ usuario/camino en la metrópoli de Guadalajara, México. Tecnura, 22(55), 51-65. https://doi. org/10.14483/22487638.13245

García Schilardi, M. E. (2017). Evaluación de la dimension operativa del transporte colectivo en el área metropolitana de Mendoza, Argentina. Perspectiva Geográfica, 22(2), 29-46. https://doi. org/10.19053/01233769.5965

González, M. (2018) Entropy and negentropy of private electric vehicles in urban systems: homeostasis of 
mobility in Mexico. DYNA, 85(206), 171-177. https:// doi.org/10.15446/dyna.v85n206.72509

Gutiérrez, A. (2010). Movilidad y acceso: Una renovación aplicada al ordenamiento territorial. Scripta Nova, 14(331). Recuperado de http://www. ub.edu/geocrit/sn/sn-331/sn-331-86.htm

Hasz, L. \& Nigri, L. (2018). Movilidades urbanas, espacios públicos y manifestaciones populares: un análisis de las protestas ocurridas en la ciudad de Belo Horizonte entre 2012 y 2015. Universitas Humanistica， 85(85). https://doi.org/10.11144/ Javeriana.uh85.muep

Hernández, J. (2018). Influencia de la estructura urbana en los patrones de movilidad cotidiana de un trabajo feminizado en la Zona Metropolitana de la Ciudad de México. Perspectiva Geográfica, 23(2), 127-147. https://doi.org/10.19053/01233769.7380

IIEG. (2015). Distribución territorial. Estadísticas. Recuperado de https://iieg.gob.mx/general. php?id=4\&idg=331

ITDP. (2013). Desarrollo orientado al transporte. México, Distrito Federal, México. Recuperado de: $\quad$ http://mexico.itdp.org/wp-content/uploads/ Desarrollo-Orientado-al-Transporte.pdf

Maynard, V., Parker, E., Yoseph-Paulus, R. \& Garcia, D. (2018). Urban planning following humanitarian crises: supporting urban communities and local governments to take the lead. Environment and Urbanization, 30(1), 265-282. https://doi. org/10.1177/0956247817732727

Mimet, A., Kerbiriou, C., Simon, L., Julien, J. F. \& Raymond, R. (2020). Contribution of private gardens to habitat availability, connectivity and conservation of the common pipistrelle in Paris. Landscape and Urban Planning, 193(1). https://doi.org/10.1016/j. landurbplan.2019.103671

Rozas P., Bonifaz J. L. \& Guerra G. (2012). El financiamiento de la infraestructura: propuestas para el desarrollo sostenible de una politica sectorial. Santiago: CEPAL.

Sotomayor, B. \& Samaniego, H. (2020). City limits in the age of smartphones and urban scaling. Computers,
Environment and Urban Systems, 79(1), 2020. https:// doi.org/10.1016/j.compenvurbsys.2019.101423

SEDESOL-CONAPO. (2012). Catálogo. Sistema Urbano Nacional 2012: México D.F. Gobierno Federal. Recuperado de http://www.conapo.gob.mx/ work/models/CONAPO/Resource/1539/1/images/ PartesIaV.pdf

SEDESOL-CONAPO-INEGI. (2012). Delimitación de las zonas metropolitanas de México 2010. Recuperado de: $\quad$ http://www.conapo.gob.mx/work/models/ CONAPO/Resource/1539/1/images/PartesIaV.pdf

Santos, G.,H., Behrendt, H. \& Teytelboym,A. (2010). Part II: Policy instruments for sustainable road transport, Research in Transportation Economics, 28(1), 46-91. https://doi.org/10.1016/j.retrec.2010.03.002

SCT. (2012). Carta topográfica del Estado de Jalisco. Secretaría de Comunicaciones y Transportes, Dirección General de Planeación. Recuperado de http://www.sct.gob.mx/fileadmin/DireccionesGrales/ DGP/Atlas/Cartografia-2014/Jalisco.pdf

Xia, C., Yeh, A.G.O. \& Zhang, A. (2020). Analyzing spatial relationships between urban land use intensity and urban vitality at street block level: A case study of five Chinese megacities. Landscape and Urban Planning, 193(1). https://doi.org/10.1016/j. landurbplan.2019.103669. 